\title{
Short-Output Universal Hash Functions and Their Use in Fast and Secure Data Authentication
}

\author{
Long Hoang Nguyen and A.W. Roscoe \\ Oxford University, Department of Computer Science \\ hn2503@gmail.com, Bill.Roscoe@cs.ox.ac.uk
}

\begin{abstract}
Message authentication codes usually require the underlining universal hash functions to have a long output so that the probability of successfully forging messages is low enough for cryptographic purposes. To take advantage of fast operation on word-size parameters in modern processors, long-output universal hashing schemes can be securely constructed by concatenating several different instances of a short-output primitive. In this paper, we describe a new method for short-output universal hash function termed digest() suitable for very fast software implementation and applicable to secure message authentication. The method possesses a higher level of security relative to other well-studied and computationally efficient short-output universal hashing schemes. Suppose that the universal hash output is fixed at one word of $b$ bits, then the collision probability of ours is $2^{1-b}$ compared to $6 \times 2^{-b}$ of $\mathrm{MMH}$, whereas $2^{-b / 2}$ of $\mathrm{NH}$ within UMAC is far away from optimality. In addition to message authentication codes, we show how short-output universal hashing is applicable to manual authentication protocols where universal hash keys are used in a very different and interesting way.
\end{abstract}

\section{Introduction}

Universal hash functions (or UHFs) first introduced by Carter and Wegman [4] have many applications in computer science, including randomised algorithms, database, cryptography and many others. A UHF takes two inputs which are a key $k$ and a message $m: h(k, m)$, and produces a fixed-length output. Normally what we require of a UHF is that for any pair of distinct messages $m$ and $m^{\prime}$ the collision probability $h(k, m)=h\left(k, m^{\prime}\right)$ is small when key $k$ is randomly chosen from its domain. In the majority of cryptographic uses, UHFs usually have long outputs so that combinatorial search is made infeasible. For example, UHFs can be used to build secure message authentication codes or MAC schemes where the intruder's ability to forge messages is bounded by the collision probability of the UHF. In a MAC, parties share a secret universal hash key and an encryption key, a message is authenticated by hashing it with the shared universal hash key and then encrypting the resulting hash. The encrypted hash value together with the message is transmitted as an authentication tag that can be validated by the verifier. We note however that our new construction presented here is applicable

A. Canteaut (Ed.): FSE 2012, LNCS 7549, pp. 326-345, 2012.

(C) International Association for Cryptologic Research 2012 
to other cryptographic uses of universal hashing, e.g., manual authentication protocols as seen later as well as non-cryptographic applications.

Since operating on short-length values of 16, 32 or 64 bits is fast and convenient in ordinary computers, long-output UHFs can be securely constructed by concatenating the results of multiple instances of short-output UHFs to increase computational efficiency. To our knowledge, a number of short-output UHF schemes have been proposed, notably MMH (Multilinear-Modular-Hashing) of Halevi and Krawczyk [8] and NH within UMAC of Black et al. [3]. We note that widely studied polynomial universal hashing schemes GHASH, PolyP and PolyQ [12] can also be designed to produce a short output. While polynomial based UHFs only require short and fixed length keys, they suffer from an unpleasant property relating to security as will be discussed later in the paper.

Our main contribution presented in Section 3 is the introduction of a new short-output UHF algorithm termed $\operatorname{digest}(k, m)$ that can be efficiently computed on any modern microprocessors. The main advantage of ours is that it provides a higher level of security regarding both collision and distribution probabilities relative to $\mathrm{MMH}$ and $\mathrm{NH}$ described in Section 4. Our digest() algorithm operates on word-size parameters via word multiplication and word addition instructions, i.e. finite fields or non-trivial reductions are excluded, because the emphasis is on high speed implementation using software.

Let us suppose that the universal hash output is fixed at one word of $b$ bits then the collision probability of ours is $2^{1-b}$ compared to $6 \times 2^{-b}$ of MMH, whereas $2^{-b / 2}$ of $\mathrm{NH}$ is much weaker in security. For clarity, the security bounds of our constructions as well as MMH and NH are independent of the length of message being hashed, which is the opposite of polynomial universal hashing schemes mentioned earlier. For multiple-word output universal hashing constructions as required in MACs, the advantage in security of ours becomes more apparent. When the universal hash output is extended to $n$ words or $n \times b$ bits for any $n \in \mathbb{N}^{*}$, then the collision probability of ours is $2^{n-n b}$ as opposed to $6^{n} \times 2^{-n b}$ of $\mathrm{MMH}$ and $2^{-n b / 2}$ of NH. There is however a trade-off between security and computational cost as illustrated by our estimated operation counts and software implementations of these constructions. On a $1 \mathrm{GHz}$ AMD Athlon processor, one version of digest() (where the collision probability $\epsilon_{c}$ is $2^{-31}$ ) achieves peak performance of 0.53 cycles/byte (or cpb) relative to $0.31 \mathrm{cpb}$ of $\mathrm{MMH}$ (for $\epsilon_{c}=$ $2^{-29.5}$ ) and $0.23 \mathrm{cpb}$ of $\mathrm{NH}$ (for $\epsilon_{c}=2^{-32}$ ). Another version of $\operatorname{digest}(k, m)$ for $\epsilon_{c}=2^{-93}$ achieves peak performance of $1.54 \mathrm{cpb}$. For comparison purpose, 12.35 $\mathrm{cpb}$ is the speed of SHA-256 recorded on our computer. A number of files that provide the software implementations in $\mathrm{C}$ programming language of $\mathrm{NH}, \mathrm{MMH}$ and our proposed constructions can be downloaded from [1] so that the reader can run them and adapt them for other uses of the schemes.

We will briefly discuss the motivation of designing as well as the elegant graphical structure of our digest() scheme which, we have only recently discovered, relates to the multiplicative universal hashing schemes of Dietzfelbinger et al. [5], Krawczyk [1] and Mansour et al. [15]. The latter algorithms are however not efficient when the input message is of a significant size. 
Although researchers from cryptographic community have mainly studied UHFs to construct message authentication codes, we would like to point out that short-output UHF on its own has found applications in manual authentication protocols [7/314/610/17/8 19 20|26]. In the new family of authentication protocols, data authentication can be achieved without the need of passwords, shared private keys as required in MACs, or any pre-existing security infrastructures such as a PKI. Instead human owners of electronic devices who seek to exchange their data authentically would need to manually compare a short string of bits that is often outputted from a UHF. Since humans can only compare short strings, the UHF ideally needs to have a short output of say 16 or 32 bits. There is however a fundamental difference in the use of universal hash keys between manual authentication protocols and message authentication codes, it will be clear in Section 5 that none of the short-output UHF schemes including ours should be used directly in the former. Thus we will propose a general framework where any short-output UHFs can be used efficiently and securely to digest a large amount of data in manual authentication protocols.

While existing universal hashing methods are already as fast as the rate information is generated, authenticated and transmitted in high-speed network traffic, one may ask whether we need another universal hashing algorithm. Besides keeping up with network traffic, as excellently explained by Black et al. [3] the goal is to use the smallest possible fraction of the CPU's cycles (so most of the machine's cycles are available for other work), by the simplest possible hash mechanism, and having the best proven bounds. This is relevant to MACs as well as manual authentication protocols where large data are hashed into a short string, and hence efficient short-output UHF constructions possessing a higher (or optimal) level of security are needed.

\section{Notation and Definitions}

We define $M, K$ and $b$ the bit length of the message, the key and the output of a universal hash function. We denote $R=\{0,1\}^{K}, X=\{0,1\}^{M}$ and $Y=\{0,1\}^{b}$.

Definition 1. 11] A $\epsilon$-balanced universal hash function, $h: R \times X \rightarrow Y$, must satisfy that for every $m \in X \backslash\{0\}$ and $y \in Y: \operatorname{Pr}_{\{k \in R\}}[h(k, m)=y] \leq \epsilon$

Many existing UHF constructions 3811 as well as our newly proposed scheme rely on (integer or matrix) multiplications of message and key, and hence nonzero input message is required; for otherwise $h(k, 0)=0$ for any key $k \in R$.

Definition 2. 1124] A $\epsilon$-almost universal hash function, $h: R \times X \rightarrow Y$, must satisfy that for every $m, m^{\prime} \in X\left(m \neq m^{\prime}\right): \operatorname{Pr}_{\{k \in R\}}\left[h(k, m)=h\left(k, m^{\prime}\right)\right] \leq \epsilon$

Since it is useful particularly in manual authentication protocols discussed later to have both the collision and distribution probabilities bounded, we combine Definitions [1] and 2 as follows. 
Definition 3. An $\epsilon_{d}$-balanced and $\epsilon_{c}$-almost universal hash function, $h: R \times$ $X \rightarrow Y$, satisfies

- for every $m \in X \backslash\{0\}$ and $y \in Y: \operatorname{Pr}_{\{k \in R\}}[h(k, m)=y] \leq \epsilon_{d}$

- for every $m, m^{\prime} \in X\left(m \neq m^{\prime}\right): \operatorname{Pr}_{\{k \in R\}}\left[h(k, m)=h\left(k, m^{\prime}\right)\right] \leq \epsilon_{c}$

\section{$3 \quad$ Integer Multiplication Construction}

We first discuss the multiplicative universal hashing algorithm of Dietzfelbinger et al. [5] which obtains a very high level of security. Although this scheme is not efficient with long input data, it strongly relates to our digest() method that makes use of word multiplication instructions.

We note that there are two other universal hashing schemes which use arithmetic that computer likes to do to increase computational efficiency, namely MMH of Halevi and Krawczyk [8] and NH of Black et al. 3]. Both of which will be compared against our construction in Section 4 .

\subsection{Multiplicative Universal Hashing}

Suppose that we want to compute a $b$-bit universal hash of a $M$-bit message, then the universal hash key $k$ is drawn randomly from $R=\left\{1,3, \ldots, 2^{M}-1\right\}$, i.e. $k$ must be odd. Dietzfelbinger et al. [5] define:

$$
h(k, m)=\left(k * m \bmod 2^{M}\right) \operatorname{div} 2^{M-b}
$$

It was proved that the collision probability of this construction is $\epsilon_{c}=2^{1-b}$ on equal length inputs [5]. While this has a simple description, for long input messages of several $\mathrm{KB}$ or $\mathrm{MB}$, such as documents and images, it will become very time consuming to compute the integer multiplication involved in this algorithm.

\subsection{Word Multiplicative Construction}

In this section, we will define and prove the security of a new short-output universal hashing scheme termed digest $(k, m)$ that can be calculated using word multiplications instead of an arbitrarily long integer multiplication as seen in Equation 1 or an example from Figure 1 .

Let us divide message $m$ into $b$-bit blocks $\left\langle m_{1}, \ldots, m_{t=M / b}\right\rangle$. An $(M+b)$ bit key $k=\left\langle k_{1}, \ldots, k_{t+1}\right\rangle$ is selected randomly from $R=\{0,1\}^{M+b}$. A $b$-bit $\operatorname{digest}(k, m)$ is defined as

$$
\operatorname{digest}(k, m)=\sum_{i=1}^{t}\left[m_{i} * k_{i}+\left(m_{i} * k_{i+1} \operatorname{div} 2^{b}\right)\right] \bmod 2^{b}
$$

Here, ${ }^{*}$ refers to a word multiplication of two $b$-bit blocks which produces a $2 b$ bit output, whereas both ' + ' and $\sum$ are additions modulo $2^{b}$. We note that (div $\left.2^{b}\right)$ is equivalent to a right shift $(>>b)$ and hence is efficient to compute. 


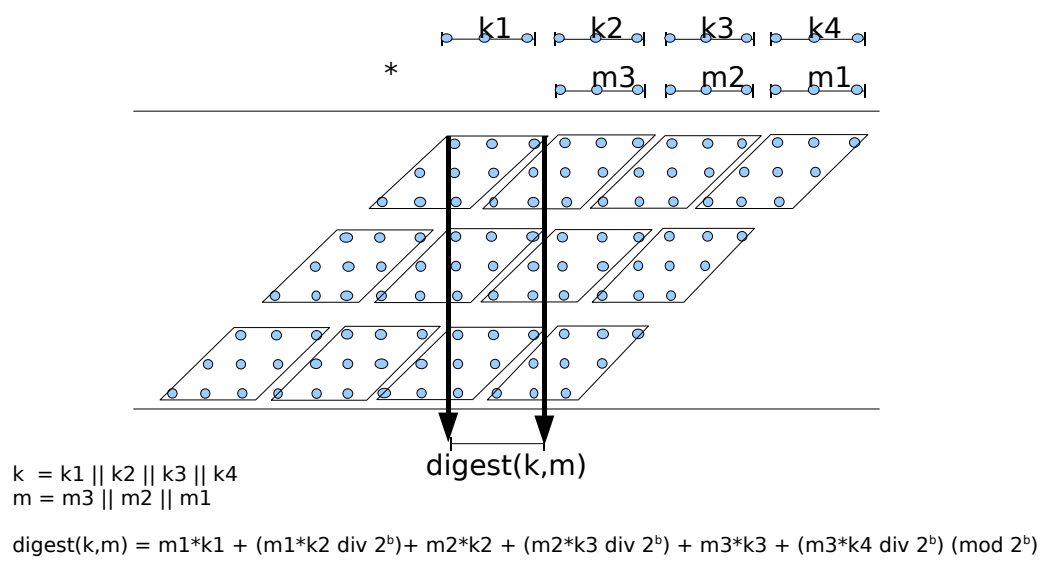

Fig. 1. A $b$-bit output digest $(k, m)$ : each parallelogram represents the expansion of a word multiplication between a $b$-bit key block and a $b$-bit message block

To see why this scheme is related to the multiplicative method of Dietzfelbinger et al. [5], one can study Figure 1] where all word multiplications involved in Equation 1 are elegantly arranged into the same shape as the overlap of the expanded multiplication between $m$ and $k 1$

Essentially what we are doing here is to obtain a short $b$-bit window in the middle of the product without computing the whole product 2 Such an idea is very similar to the SQUASH hash function of Shamir [23] that produces an excellent numeric approximation of the $b$ middle bits by computing a longer window of $b+u$ bits with $u$ additional lower order bits so that the full effect of the carry bits is significantly restored. There are however three crucial differences between ours and SQUASH: (1) we do not need to compute the extra $u$ lower order words or bits, (2) we partially ignore the carry between words, and (3) as opposed to SQUASH, the security of digest() does not rely on the Rabin public key encryption scheme [23]. The first two of these make ours much faster in computation.

Operation Count. To give an estimated operation count for an implementation of digest(), which will be subsequently compared against universal hashing schemes $\mathrm{MMH}$ and $\mathrm{NH}$, we consider a machine with the same properties as one used by Halevi and Krawczyk [8]

\footnotetext{
${ }^{1}$ If we further ignore the effect of the carry in (word) multiplications of both digest() and the scheme of Dietzfelbinger et al. then they become very similar to the Toeplitz matrix based construction of Krawczyk 11 and Mansour et al. 15. Such a carry-less multiplication instruction is available in a new Intel processor [2].

2 This idea was first reported in our patent application 21 dated back to 2006 .

3 The same operation count given here is applicable to a $(2 b=64)$-bit machine. In the latter, a multiplication of two 32-bit unsigned integer is stored in a single 64-bit register, and High and Low are the upper and lower 32-bit halves of the register.
} 
- $(b=32)$-bit machine and arithmetic operations are done in registers.

- A multiplication of two 32-bit integers yields a 64-bit result that is stored in 2 registers.

A pseudo-code for digest() on such machine may be as follows. For a ' $\mathrm{C}$ ' implementation, please see [1].

digest(key, msg)

1. $\quad$ Sum $=0$

2. $\quad$ load key[1]

3. for $i=1$ to $t$

4. $\quad$ load $m s g[i]$

5. $\quad \quad \quad$ load key $[i+1]$

6. $\quad\langle$ High 1, Low 1$\rangle=m s g[i] * \operatorname{key}[i]$

7. $\quad\langle$ High $2, \operatorname{Low} 2\rangle=\operatorname{msg}[i] * \operatorname{key}[i+1]$

8. $\quad$ Sum $=$ Sum + Low $1+$ High 2

9. return Sum

This consists of $2 t=2 M / b$ word multiplications (MULT) and $2 t=2 M / b$ addition modulo $2^{b}$ (ADD). That is each message-word requires 1 MULT and 2 ADD operations. As in 8], a MULT/ADD operation should include not only the actual arithmetic instruction but also loading the message- and key-words to registers and/or loop handling.

The following theorem shows that the switch from a single (arbitrarily long) multiplication of Dietfelbinger et al. into word multiplications of digest() does not weaken the security of the construction. Namely the same collision probability of $2^{1-b}$ is retained while optimality in distribution is achieved. Moreover this change not only greatly increases computational efficiency but also removes the restriction of odd universal hash key as required in Dietfelbinger et al.

Theorem 1. For any $t, b \geq 1$, digest () of Equation 1 satisfies Definition 3 with the distribution probability $\epsilon_{d}=2^{-b}$ and the collision probability $\epsilon_{c}=2^{1-b}$ on equal length inputs.

Proof. We first consider the collision property. For any pair of distinct messages of equal length: $m=m_{1} \cdots m_{t}$ and $m^{\prime}=m_{1}^{\prime} \cdots m_{t}^{\prime}$, without loss of generality we assume that $m_{1}>m_{1}^{\prime} 4$ A digest collision is equivalent to:

$$
\sum_{i=1}^{t}\left[m_{i} * k_{i}+\left(m_{i} * k_{i+1} \operatorname{div} 2^{b}\right)\right]=\sum_{i=1}^{t}\left[m_{i}^{\prime} * k_{i}+\left(m_{i}^{\prime} * k_{i+1} \operatorname{div} 2^{b}\right)\right] \quad\left(\bmod 2^{b}\right)
$$

There are two possibilities as follows.

\footnotetext{
4 Please note that when $m_{i}=m_{i}^{\prime}$ for all $i \in\{1, \ldots, j\}$ then in the following calculation we will assume that $m_{j+1}>m_{j+1}^{\prime}$.
} 
WHEN $m_{1}-m_{1}^{\prime}$ is odd. The above equality can be rewritten as

$$
\left(m_{1}-m_{1}^{\prime}\right) k_{1}=y \quad\left(\bmod 2^{b}\right)
$$

where

$$
\begin{aligned}
y= & \left(m_{1}^{\prime} k_{2} \operatorname{div} 2^{b}\right)-\left(m_{1} k_{2} \operatorname{div} 2^{b}\right)+ \\
& \sum_{i=2}^{t}\left[\left(m_{i}^{\prime}-m_{i}\right) * k_{i}+\left(m_{i}^{\prime} * k_{i+1} \operatorname{div} 2^{b}\right)-\left(m_{i} * k_{i+1} \operatorname{div} 2^{b}\right)\right]
\end{aligned}
$$

We note that $y$ depends only on keys $k_{2}, \ldots, k_{t+1}$, and hence we fix $k_{2}$ through $k_{t+1}$ in our analysis. Since $m_{1}-m_{1}^{\prime}$ is odd, i.e. $m_{1}-m_{1}^{\prime}$ and $2^{b}$ are co-prime, there is at most one value of $k_{1}$ satisfying Equation 2, The collision probability in this case is therefore $\epsilon_{c}=2^{-b}<2^{1-b}$.

WHEN $m_{1}-m_{1}^{\prime}$ is even. A digest collision can be rewritten as

$$
\left(m_{1}-m_{1}^{\prime}\right) k_{1}+\left(m_{1} k_{2} \operatorname{div} 2^{b}\right)-\left(m_{1}^{\prime} k_{2} \operatorname{div} 2^{b}\right)+\left(m_{2}-m_{2}^{\prime}\right) k_{2}=y \quad\left(\bmod 2^{b}\right)
$$

where

$$
\begin{aligned}
y= & \left(m_{2}^{\prime} k_{3} \operatorname{div} 2^{b}\right)-\left(m_{2} k_{3} \operatorname{div} 2^{b}\right)+ \\
& \sum_{i=3}^{t}\left[\left(m_{i}^{\prime}-m_{i}\right) * k_{i}+\left(m_{i}^{\prime} * k_{i+1} \operatorname{div} 2^{b}\right)-\left(m_{i} * k_{i+1} \operatorname{div} 2^{b}\right)\right]\left(\bmod 2^{b}\right)
\end{aligned}
$$

We note that $y$ depends only on keys $k_{3}, \ldots, k_{t+1}$. If we fix $k_{3}$ through $k_{t+1}$ in our analysis, we need to find the number of pairs $\left(k_{1}, k_{2}\right)$ such that Equation 3 is satisfied. We arrive at

$$
\epsilon_{c}=\operatorname{Prob}_{\left\{k_{1}, k_{2}\right\}}\left[\left(m_{1}-m_{1}^{\prime}\right) k_{1}+\left(m_{1} k_{2} \operatorname{div} 2^{b}\right)-\left(m_{1}^{\prime} k_{2} \operatorname{div} 2^{b}\right)+\left(m_{2}-m_{2}^{\prime}\right) k_{2}=y\right]
$$

Let us define

$$
\begin{aligned}
& m_{1} k_{2}=u 2^{b}+v \\
& m_{1}^{\prime} k_{2}=u^{\prime} 2^{b}+v^{\prime}
\end{aligned}
$$

Since we assumed $m_{1}>m_{1}^{\prime}$, we have $u \geq u^{\prime}$ and $\left(m_{1}-m_{1}^{\prime}\right) k_{2}=\left(u-u^{\prime}\right) 2^{b}+v-v^{\prime}$.

- When $v \geq v^{\prime}:\left(m_{1} k_{2} \operatorname{div} 2^{b}\right)-\left(m_{1}^{\prime} k_{2} \operatorname{div} 2^{b}\right)=\left(m_{1}-m_{1}^{\prime}\right) k_{2} \operatorname{div} 2^{b}$

- When $v<v^{\prime}:\left(m_{1} k_{2} \operatorname{div} 2^{b}\right)-\left(m_{1}^{\prime} k_{2} \operatorname{div} 2^{b}\right)=\left[\left(m_{1}-m_{1}^{\prime}\right) k_{2} \operatorname{div} 2^{b}\right]+1$

Let $c=m_{1}-m_{1}^{\prime}$ and $d=m_{2}-m_{2}^{\prime}\left(\bmod 2^{b}\right)$, we then have $1 \leq c<2^{b}$ and:

$$
\epsilon_{c} \leq p_{1}+p_{2}
$$

where

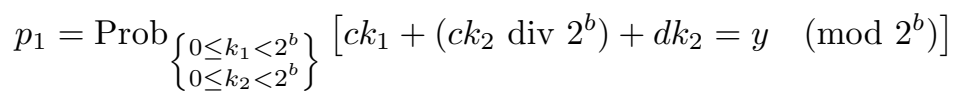


and

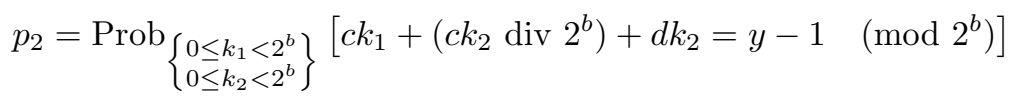

Using Lemma 1, we have $p_{1}, p_{2} \leq 2^{-b}$, and thus $\epsilon_{c} \leq 2^{1-b}$.

As regards distribution, since $m=m_{1} \cdots m_{t}>0$ as specified in Definition 3 . without loss of generality we assume that $m_{1} \geq 1$. If we fix $k_{3}$ through $k_{t+1}$ and for any $y \in\left\{0, \ldots, 2^{b}-1\right\}$, then the distribution probability $\epsilon_{d}$ is equivalent to:

$$
\epsilon_{d}=\operatorname{Prob}_{\left\{\begin{array}{l}
0 \leq k_{1}<2^{b} \\
0 \leq k_{2}<2^{b}
\end{array}\right.}\left[m_{1} k_{1}+\left(m_{1} k_{2} \operatorname{div} 2^{b}\right)+m_{2} k_{2}=y \quad\left(\bmod 2^{b}\right)\right]
$$

Since $1 \leq m_{1}<2^{b}$, we can use Lemma 1 to deduce that $\epsilon_{d}=2^{-b}$.

Lemma 1. Let $1 \leq c<2^{b}$ and $0 \leq d<2^{b}$, then for any $y \in\left\{0, \ldots, 2^{b}-1\right\}$ we have

$$
\operatorname{Prob}_{\left\{\begin{array}{l}
0 \leq k_{1}<2^{b} \\
0 \leq k_{2}<2^{b}
\end{array}\right\}}\left[c k_{1}+\left(c k_{2} \operatorname{div} 2^{b}\right)+d k_{2}=y \quad\left(\bmod 2^{b}\right)\right]=2^{-b}
$$

Proof. We write $c=s 2^{l}$ with $s$ odd and $0 \leq l<b$. Since $s$ and $2^{b}$ are co-prime, there exist a unique inverse modulo $2^{b}$ of $s$, we call it $s^{-1}$. Our equation now becomes:

$$
2^{l} s k_{1}+\left(2^{l} s k_{2} \operatorname{div} 2^{b}\right)+d s^{-1} s k_{2}=y \quad\left(\bmod 2^{b}\right)
$$

Let $s k_{1}=\gamma\left(\bmod 2^{b-l}\right)$ and $s k_{2}=\alpha 2^{b-l}+\beta\left(\bmod 2^{b}\right)$, we then have $0 \leq \gamma<$ $2^{b-l}$ and $0 \leq \alpha<2^{l}$. The above equation becomes:

$$
\begin{array}{cc}
2^{l} \gamma+\alpha+d s^{-1}\left(\alpha 2^{b-l}+\beta\right)=y & \left(\bmod 2^{b}\right) \\
2^{l} \gamma+\alpha\left(1+d s^{-1} 2^{b-l}\right)+\beta d s^{-1}=y & \left(\bmod 2^{b}\right) \\
2^{l} \gamma+\alpha x=z \quad\left(\bmod 2^{b}\right) &
\end{array}
$$

where $x=1+d s^{-1} 2^{b-l}\left(\bmod 2^{b}\right)$ which is always odd because $l<b$, and $z=y-\beta d s^{-1}\left(\bmod 2^{b}\right)$. Since $z$ is independent of $\gamma$ and $\alpha$, we fix $z$ in our analysis. We can then use Lemma 2 to derive that there is a unique pair $(\gamma, \alpha)$ satisfying the above equation.

Since $0 \leq \gamma<2^{b-l}$ and $0 \leq \alpha<2^{l}, \gamma$ and $\alpha$ together determine $b$ bits of the combination of $k_{1}$ and $k_{2}$. Consequently there are at most $2^{b}$ different pairs $\left(k_{1}, k_{2}\right)$ satisfying the condition that we require in this lemma.

Lemma 2. Let $0 \leq l<b$ and $x \in\left\{1,3, \ldots, 2^{b}-1\right\}$ then for any $z \in$ $\left\{0, \ldots, 2^{b}-1\right\}$ there is a unique pair $(\gamma, \alpha)$ such that $0 \leq \gamma<2^{b-l}, 0 \leq \alpha<2^{l}$, and $2^{l} \gamma+\alpha x=z\left(\bmod 2^{b}\right)$.

Proof. If there exist two distinct pairs $(\gamma, \alpha)$ and $\left(\gamma^{\prime}, \alpha^{\prime}\right)$ satisfying this condition, then

$$
2^{l} \gamma+\alpha x=2^{l} \gamma^{\prime}+\alpha^{\prime} x=z \quad\left(\bmod 2^{b}\right)
$$


which implies that

$$
2^{l}\left(\gamma-\gamma^{\prime}\right)=\left(\alpha^{\prime}-\alpha\right) x \quad\left(\bmod 2^{b}\right)
$$

This leads to two possibilities.

- When $\alpha^{\prime}=\alpha$ then $2^{l}\left(\gamma-\gamma^{\prime}\right)=0$, which means that $2^{b-l} \mid\left(\gamma-\gamma^{\prime}\right)$. The latter is impossible because $0 \leq \gamma, \gamma^{\prime}<2^{b-l}$ and $\gamma \neq \gamma^{\prime}$.

- When $\alpha^{\prime} \neq \alpha$ and since $x$ is odd, we must have $2^{l} \mid\left(\alpha^{\prime}-\alpha\right)$. This is also impossible because $0 \leq \alpha, \alpha^{\prime}<2^{l}$.

REMARKS. The bound given by Theorem 1 for the distribution probability $\left(\epsilon_{d}=2^{-b}\right)$ is tight: let $m=0^{b-1} 1$ and any $y$ and note that any key $k=k_{1} k_{2}$ with $k_{1}=y$ satisfying this equation $\operatorname{digest}(k, m)=y$. The bound given by Theorem 1 for the collision probability $\epsilon_{c}=2^{1-b}$ also appears to be tight, i.e. it cannot be reduced to $2^{-b}$. To verify this bound, we have implemented exhaustive tests on single-word messages with small value of $b$. For example, when $b=7$, we look at all possible pairs of two different $(b=7)$-bit messages in combination with all $(2 b=14)$-bit keys, the obtained collision probability is $2^{-7} \times 1.875$.

We end this section by pointing out that truncation is secure in this digest construction. For any $b^{\prime} \in\{1, \ldots, b-1\}$, we define

$$
\operatorname{trunc}_{b^{\prime}}(\operatorname{digest}(k, m))=\sum_{i=1}^{t}\left[m_{i} * k_{i}+\left(m_{i} * k_{i+1} \operatorname{div} 2^{b}\right)\right] \bmod 2^{b^{\prime}}
$$

where trunc $b^{\prime}()$ takes the first $b^{\prime}$ least significant bits of the input. We then have the following theorem whose proof is very similar to the proof of Theorem 1, and hence it is not given here.

Theorem 2. For any $n, t \geq 1, b \geq 1$ and any integer $b^{\prime} \in\{1, \ldots, b-1\}$, trunc $_{b^{\prime}}\left(\right.$ digest $\left._{())}\right)$of Equation 4 satisfies Definition 3 with the distribution probability $\epsilon_{d}=2^{-b^{\prime}}$ and the collision probability $\epsilon_{c}=2^{1-b^{\prime}}$ on equal length inputs.

\subsection{Extending $\operatorname{digest}()$}

If we want to use digest functions as the main ingredient of a message authentication code, we need to reduce the collision probability without increasing the word bitlength $b$ that is dictated by architecture characteristics. One possibility is to hash our message with several random and independent keys, and concatenate the results. If we concatenate the results from $n$ independent instances of the digest function, the collision probability drops from $2^{1-b}$ to $2^{n-n b}$. This solution however requires $n$ times as much key material.

A much better and well-studied approach is to use the Toeplitz-extension: given one key we left shift the key by one word to get the next key and digest again. The resulting construction is called digest $_{M W}()$, where $M W$ stands 


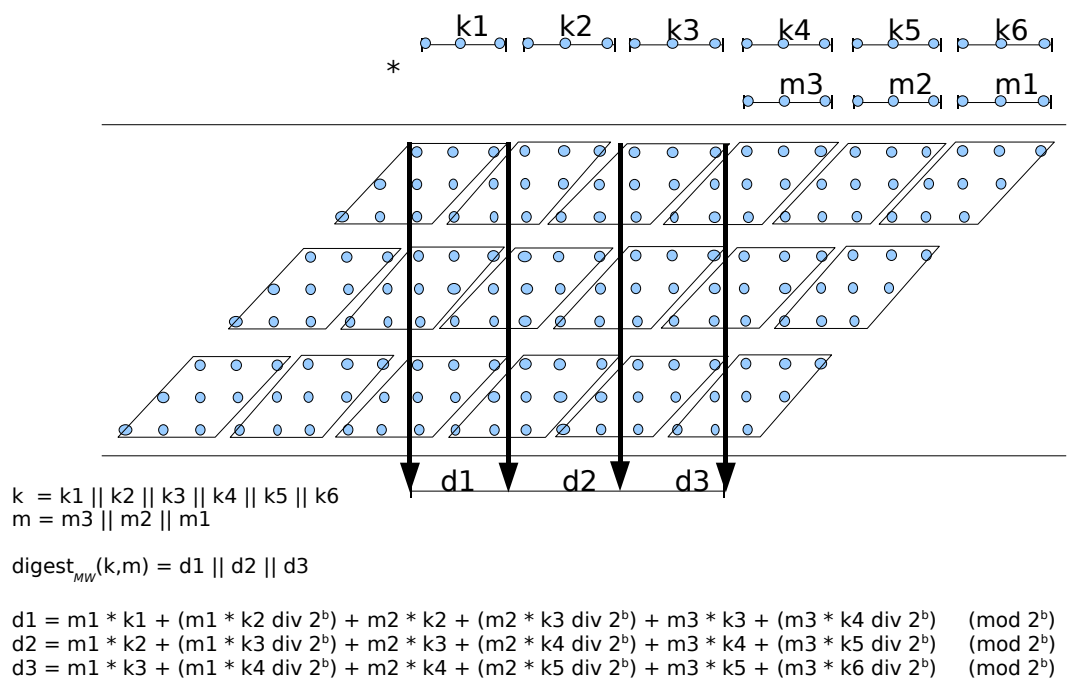

Fig. 2. A 3b-bit (or three-word) output $\operatorname{digest}_{M W}(k, m)$ : each parallelogram represents the expansion of a word multiplication between a $b$-bit key block and a $b$-bit message block

for multiple-word output. The structure of $\operatorname{digest}_{M W}()$ is again graphically illustrated by an example in Figure 2 that shows a similar connection between digest $_{M W}()$ and the multiplicative hashing scheme of Dietfelbinger et al.

We define a $n$-blocks or $(n \times b)$-bit output $\operatorname{digest}_{M W}(k, m)$ as follows. We still divide $m$ into $b$-bit blocks $\left\langle m_{1}, \ldots, m_{t=M / b}\right\rangle$. However, an $(M+b n)$-bit key $k=\left\langle k_{1}, \ldots, k_{t+n}\right\rangle$ will be chosen randomly from $R=\{0,1\}^{M+b n}$ to compute a $n b$-bit digest.

For all $i \in\{1, \ldots, n\}$, we then define:

$$
d_{i}=\operatorname{digest}\left(k_{i \cdots t+i}, m\right)=\sum_{j=1}^{t}\left[m_{j} k_{i+j-1}+\left(m_{j} k_{i+j} \operatorname{div} 2^{b}\right)\right] \bmod 2^{b}
$$

And

$$
\operatorname{digest}_{M W}(k, m)=\left\langle d_{1} \cdots d_{n}\right\rangle
$$

The following theorem and its proof show that $\operatorname{digest}_{M W}()$ enjoys the best bound for both collision and distribution probabilities that one could hope for.

Theorem 3. For any $n, t \geq 1$ and $b \geq 1$, digest ${ }_{M W}()$ satisfies Definition 3with the distribution probability $\epsilon_{d}=2^{-n b}$ and the collision probability $\epsilon_{c}=2^{n-n b}$ on equal length inputs.

Proof. We first consider the collision property of a digest function. For any pair of distinct messages of equal length: $m=m_{1} \cdots m_{t}$ and $m^{\prime}=m_{1}^{\prime} \cdots m_{t}^{\prime}$, without loss of generality we assume that $m_{1}>m_{1}^{\prime}$. Please note that when $t=1$ or 
$m_{i}=m_{i}^{\prime}$ for all $i \in\{1, \ldots, t-1\}$ then in the following calculation we will assume that $m_{t+1}=m_{t+1}^{\prime}=0$.

For $i \in\{1, \ldots, n\}$, we define Equality $E_{i}$ as

$E_{i}: \sum_{j=1}^{t}\left[m_{j} k_{i+j-1}+\left(m_{j} k_{i+j} \operatorname{div} 2^{b}\right)\right]=\sum_{j=1}^{t}\left[m_{j}^{\prime} k_{i+j-1}+\left(m_{j}^{\prime} k_{i+j} \operatorname{div} 2^{b}\right)\right] \bmod 2^{b}$

and thus the collision probability is: $\epsilon_{c}=\operatorname{Prob}_{\{k \in R\}}\left[E_{1} \wedge \cdots \wedge E_{n}\right]$.

Since all arithmetic operations are done over modulo $2^{b}$, for simplicity we ignore $\left(\bmod 2^{b}\right)$ in our notation.

WHEN $m_{1}-m_{1}^{\prime}$ is odd. We proceed by proving that for all $i \in\{1, \ldots, n\}$

$$
\operatorname{Prob}\left[E_{i} \text { is true } \mid E_{i+1}, \ldots, E_{n} \text { are true }\right] \leq 2^{-b}
$$

For Equality $E_{n}$, the claim is satisfied due to Theorem[1. We notice that Equalities $E_{i+1}$ through $E_{n}$ depend only on keys $k_{i+1}, \ldots, k_{n+t}$, whereas Equality $E_{i}$ depends also on key $k_{i}$. Fix $k_{i+1}$ through $k_{n+t}$ such that Equalities $E_{i+1}$ through $E_{n}$ are satisfied. We prove that there is at most one value of $k_{i}$ satisfying $E_{i}$. To achieve this we let

$$
\begin{aligned}
z= & \left(m_{1}^{\prime} k_{i+1} \operatorname{div} 2^{b}\right)-\left(m_{1} k_{i+1} \operatorname{div} 2^{b}\right)+ \\
& \sum_{j=2}^{t}\left[\left(m_{j}^{\prime}-m_{j}\right) k_{i+j-1}+\left(m_{j}^{\prime} k_{i+j} \operatorname{div} 2^{b}\right)-\left(m_{j} k_{i+j} \operatorname{div} 2^{b}\right)\right]
\end{aligned}
$$

we then rewrite Equality $E_{i}$ as

$$
\left(m_{1}-m_{1}^{\prime}\right) k_{i}=z
$$

Since we assumed $m_{1}-m_{1}^{\prime}$ is odd, there is at most one value of $k_{i}$ satisfying this equation.

WHEN $m_{1}-m_{1}^{\prime}$ is even. We write $m_{1}-m_{1}^{\prime}=2^{l} s$ with $s$ odd and $0<l<b$, and $s^{\prime}=\left(m_{2}^{\prime}-m_{2}\right) s^{-1}$. We further denote $s k_{i}=x_{i} 2^{b-l}+y_{i}$ for $i \in\{1, \ldots, n+t\}$, where $0 \leq x_{i}<2^{l}$ and $0 \leq y_{i}<2^{b-l}$.

For $i \in\{1, \ldots, n\}$, if we define $b_{i} \in\{0,1\}$ and

$$
\begin{aligned}
f\left(y_{i}, x_{i+1}\right)= & 2^{l} y_{i}+x_{i+1}\left[\left(m_{2}-m_{2}^{\prime}\right) s^{-1} 2^{b-l}+1\right] \\
g\left(k_{i+2}, \ldots, k_{i+t}\right)= & \left(m_{2}^{\prime} k_{i+2} \operatorname{div} 2^{b}\right)+\sum_{j=3}^{t}\left[m_{j}^{\prime} k_{i+j-1}+\left(m_{j}^{\prime} k_{i+j} \operatorname{div} 2^{b}\right)\right]- \\
& \left(m_{2} k_{i+2} \operatorname{div} 2^{b}\right)-\sum_{j=3}^{t}\left[m_{j} k_{i+j-1}+\left(m_{j} k_{i+j} \operatorname{div} 2^{b}\right)\right]
\end{aligned}
$$


then, using similar trick as in the proof of Lemma 1, Equality $E_{i}$ can be rewritten as

$$
\begin{aligned}
\left(m_{1}-m_{1}^{\prime}\right) k_{i}+\left(\left(m_{1}-m_{1}^{\prime}\right) k_{i+1} \operatorname{div} 2^{b}\right)+\left(m_{2}-m_{2}^{\prime}\right) k_{i+1} & =g\left(k_{i+2}, \ldots, k_{i+t}\right)-b_{i} \\
2^{l} s k_{i}+\left(2^{l} s k_{i+1} \operatorname{div} 2^{b}\right)+\left(m_{2}-m_{2}^{\prime}\right) s^{-1} s k_{i+1} & =g\left(k_{i+2}, \ldots, k_{i+t}\right)-b_{i} \\
2^{l} y_{i}+x_{i+1}+\left(m_{2}-m_{2}^{\prime}\right) s^{-1}\left(x_{i+1} 2^{b-l}+y_{i+1}\right) & =g\left(k_{i+2}, \ldots, k_{i+t}\right)-b_{i}
\end{aligned}
$$

Rearranging gives

$$
\begin{aligned}
2^{l} y_{i}+x_{i+1}\left[\left(m_{2}-m_{2}^{\prime}\right) s^{-1} 2^{b-l}+1\right] & =s^{\prime} y_{i+1}-b_{i}+g\left(k_{i+2}, \ldots, k_{i+t}\right) \\
f\left(y_{i}, x_{i+1}\right) & =s^{\prime} y_{i+1}-b_{i}+g\left(k_{i+2}, \ldots, k_{i+t}\right)
\end{aligned}
$$

Putting Equalities $E_{1}$ through $E_{n}$ together, we have

$$
\begin{aligned}
E_{1}: f\left(y_{1}, x_{2}\right) & =s^{\prime} y_{2}-b_{1}+g\left(k_{3}, \ldots, k_{1+t}\right) \\
E_{2}: f\left(y_{2}, x_{3}\right) & =s^{\prime} y_{3}-b_{2}+g\left(k_{4}, \ldots, k_{2+t}\right) \\
E_{3}: f\left(y_{3}, x_{4}\right) & =s^{\prime} y_{4}-b_{3}+g\left(k_{5}, \ldots, k_{3+t}\right) \\
\vdots & \vdots \\
E_{n-1}: f\left(y_{n-1}, x_{n}\right) & =s^{\prime} y_{n}-b_{n-1}+g\left(k_{n+1}, \ldots, k_{n+t-1}\right) \\
E_{n}: f\left(y_{n}, x_{n+1}\right) & =s^{\prime} y_{n+1}-b_{n}+g\left(k_{n+2}, \ldots, k_{n+t}\right)
\end{aligned}
$$

We fix $k_{n+2}$ through $k_{t+n}$. We note that there are $2^{b-t}$ values for $y_{n+1}$ and two values for $b_{n}$. For each pair $\left(y_{n+1}, b_{n}\right)$ there is a unique pair $\left(y_{n}, x_{n+1}\right)$ satisfying Equality $E_{n}$ due to Lemma 2. Similarly, for each tuple $\left\langle y_{n}, k_{n+1}, b_{n-1}, b_{n}\right\rangle$ there is also a unique pair $\left(y_{n-1}, x_{n}\right)$ satisfying Equality $E_{n-1}$. We will continue this process until we reach the pair $\left(y_{1}, x_{2}\right)$ in Equality $E_{1}$. Since Equalities $E_{1}$ through $E_{n}$ do not depend on $x_{1}$ and there are $2^{l}$ values for $x_{1}$, there will be at most $2^{l} 2^{n} 2^{b-l}=2^{n+b}$ different tuples $\left\langle k_{1} \cdots k_{n+1}\right\rangle$ satisfying Equalities $E_{1}$ through $E_{n}$. And thus the collision probability $\epsilon_{c}=2^{n+b} / 2^{(n+1) b}=2^{n-n b}$.

Similar argument also leads to our bound on the distribution probability $\epsilon_{d}=$ $2^{-n b}$

REMARKS. Even though Theorems 1 and 3 address the collision property, their proofs can be easily adapted to show that our constructions are also $\epsilon_{c}$-almost$\Delta$-universal [8] as in the case of the MMH scheme considered in the next section. The latter property requires that for every $m, m^{\prime} \in X$ where $m \neq m^{\prime}$ and $a \in Y$ : $\operatorname{Pr}_{\{k \in R\}}\left[\operatorname{digest}(k, m)-\operatorname{digest}\left(k, m^{\prime}\right)=a\right] \leq \epsilon_{c}$.

Operation Count. The advantage of this scheme is the ability to reuse the result of each word multiplication in the computation of two adjacent digest output words as seen in Figure 2 and the following pseudo-code, e.g. the multiplication $m_{1} k_{2}$ is instrumental in the computation of both $d_{1}$ and $d_{2}$. Using the same machine as specified in subsection 3.2, each message-word therefore requires $(n+1)$ MULT and $2 n$ ADD operations. 
A pseudo-code for digest $_{M W}()$ on such machine may be as follows

digest $_{M W}(k e y, m s g)$

1. For $i=1$ to $n$

2. $d[i]=0$

3. load key $[i]$

4. For $j=1$ to $t$

5. $\quad$ load $m s g[j]$

6. $\quad \operatorname{load} k e y[j+n]$

7. $\langle$ High $[0], \operatorname{Low}[0]\rangle=\operatorname{msg}[j] * k e y[j]$

8. $\quad$ For $i=1$ to $n$

9. $\quad\langle\operatorname{High}[i], \operatorname{Low}[i]\rangle=\operatorname{msg}[j] * \operatorname{key}[j+i]$

10. $\quad d[i]=d[i]+\operatorname{Low}[i-1]+\operatorname{High}[i]$

11. $\operatorname{return}\langle d[1] \cdots d[n]\rangle$

\section{Comparative Analysis}

In this section, we mainly compare our new digest scheme against well-studied universal hashing algorithms MMH of Halevi and Krawczyk 8] and NH of Black et al. 3] described in Subsections 4.1 and 4.2 respectively. Since $\operatorname{digest()~can~}$ be extended to produce multiple-word output as in the case of $\mathrm{MMH}$ and $\mathrm{NH}$ to build MACs, our analysis consider both single- and multiple-word output schemes. We note that $\mathrm{NH}$ is the building block of not only UMAC but also UHASH16 and UHASH32 [3]. For completeness, we will discuss another widely studied UHF family based on polynomial over finite field, e.g. GHASH, PolyP, PolyQ and PolyR [12. While the polynomial universal hashing schemes only require short keys, they suffer from two unpleasant properties: (1) the collision probability decreases linearly with the message length, and (2) they are less efficient, especially in software implementation, than our digest functions as well as $\mathrm{MMH}$ and $\mathrm{NH}$ due to the involved modular arithmetic operations.

The properties of the three main schemes - MMH, NH and digest() - are summarised in Table 1 where the upper and lower halves correspond to singleword ( $b$ bits) and respectively multiple-word ( $n b$ bits) output schemes for any $n \geq$ 1. This table indicates that the security level obtained in our digest algorithm is higher than both $\mathrm{MMH}$ and $\mathrm{NH}$ with respect to the same output length. In particular, the collision probability of digest() is a third of $\mathrm{MMH}$, while $\mathrm{NH}$ must double the output length to achieve the same order of security. For multipleword output schemes, this advantage in security of our proposed digest algorithm becomes even more significant as seen in the lower half of Table 1.

We end this section by providing implementation results in Table 2 of Section 4.3. As described earlier, $\mathrm{C}$ files which contain the implementations of $\mathrm{NH}$, $\mathrm{MMH}$ and digest() as well as their multiple-word output versions can be downloaded from [1] which allows readers to test the speed of the constructions. 
Table 1. A summary on the main properties of digest(), MMH and NH. MULT operates on $b$-bit inputs, whereas ADD operates on inputs of either $b$ or $2 b$ bits.

\begin{tabular}{|l|c|c|c|c|c|c|}
\hline Scheme & Key length & MULTs/word & ADDs/word & $\epsilon_{c}$ & $\epsilon_{d}$ & Output bitlength \\
\hline digest & $M+b$ & 2 & 2 & $2^{1-b}$ & $2^{-b}$ & $b$ \\
MMH & $M$ & 1 & 1 & $6 \times 2^{-b}$ & $2^{2-b}$ & $b$ \\
NH & $M$ & $1 / 2$ & $3 / 2$ & $2^{-b}$ & $2^{-b}$ & $2 b$ \\
\hline & & & & & & \\
\hline digest $_{M W}$ & $M+n b$ & $n+1$ & $2 n$ & $2^{n-n b}$ & $2^{-n b}$ & $n b$ \\
MMH $_{M W}$ & $M+(n-1) b$ & $n$ & $n$ & $6^{n} \times 2^{-n b}$ & $2^{2 n-n b}$ & $n b$ \\
$\mathrm{NH}_{M W}$ & $M+2(n-1) b$ & $n / 2$ & $3 n / 2$ & $2^{-n b}$ & $2^{-n b}$ & $2 n b$ \\
\hline
\end{tabular}

\section{1 $\mathrm{MMH}$}

Fix a prime number $p \in\left[2^{b}, 2^{b}+2^{b / 2}\right]$. The $b$-bit output MMH universal hash function is defined for any $k=k_{1}, \ldots, k_{t}$ and $m=m_{1}, \ldots, m_{t}$ as follows

$$
\operatorname{MMH}(k, m)=\left[\left[\left[\sum_{i=1}^{t} m_{i} * k_{i}\right] \bmod 2^{2 b}\right] \bmod p\right] \bmod 2^{b}
$$

It was proved in [8] that the collision probability of MMH is $\epsilon_{c}=6 \times 2^{-b}$ as opposed to only $2^{1-b}$ of digest(). By using the same proof technique presented in [8], it is also not hard to show that the distribution probability of $\mathrm{MMH}$ is $\epsilon_{d}=2^{2-b}$, as opposed to $2^{-b}$ of digest() 5

For single-word output, each message word in MMH requires $1(b \times b)$ MULT and 1 ADD modulo $2^{2 b}$. We note however that this does not include the cost of the final reduction modulo $p$. For $n$-word output MMH, using "the Toeplitz matrix approach", the scheme is defined as

$$
\operatorname{MMH}_{M W}(k, m)=\operatorname{MMH}\left(k_{1 \cdots t}, m\right)\left\|\operatorname{MMH}\left(k_{2 \cdots t+1}, m\right)\right\| \cdots \| \operatorname{MMH}\left(k_{n \cdots t+n-1}, m\right)
$$

$\mathrm{MMH}_{M W}$ obtains $\epsilon_{c}=6^{n} 2^{-n b}$ and $\epsilon_{d}=2^{2 n-n b}$, which are considerably weaker than digest $_{M W}()\left(\epsilon_{c}=2^{n-n b}, \epsilon_{d}=2^{-n b}\right)$.

\section{$4.2 \mathrm{NH}$}

The $2 b$-bit output NH universal hash function is defined for any $k=k_{1}, \ldots, k_{t}$ and $m=m_{1}, \ldots, m_{t}$, where $t$ is even, as follows

$$
\mathrm{NH}(k, m)=\sum_{i=1}^{t / 2}\left(k_{2 i-1}+m_{2 i-1}\right)\left(k_{2 i}+m_{2 i}\right) \bmod 2^{2 b}
$$

5 There is a Square Hash variant of MMH introduced by Etzel et al. 6] that is defined as follows: $\operatorname{SQH}(k, m)=\left[\sum_{i=1}^{t}\left(\left(m_{i}+k_{i}\right) \bmod 2^{b}\right)^{2}\right] \bmod p$. The collision probability $\epsilon_{c}$ of SQH is $2^{1-b}$. While squaring an integer is more efficient than multiplication, $\mathrm{SQH}$ is not really faster than MMH because the summation of the squares does not fit into $2 b$ bits and hence extra words are required to store and add when long data are hashed. Etzel et al. then optimise the implementation by ignoring the carry bits in the computation, but this makes the collision probability bound bigger than $2^{1-b}$. 
The downside of $\mathrm{NH}$ relative to $\mathrm{MMH}$ and our digest method is the level of security obtained, namely with a $2 b$-bit output, which is twice the length of both digest() and $\mathrm{MMH}, \mathrm{NH}$ was shown to have the collision probability $\epsilon_{c}=2^{-b}$ and the distribution probability $\epsilon_{d}=2^{-b}$, which are far from optimality. Its computational cost is however lower than the other twos, i.e. each message-word requires only $1 / 2(b \times b)$ MULT, 1 ADD modulo $2^{b}$, and $1 / 2$ ADD modulo $2^{2 b}$.

For $2 n$-word output, also using "the Toeplitz matrix approach", we have $\epsilon_{c}=$ $2^{-n b}$ and $\epsilon_{d}=2^{-n b}$. Each message-word requires $n / 2$ MULT and $3 n / 2$ ADD operations as seen below.

$$
\mathrm{NH}_{M W}(k, m)=\mathrm{NH}\left(k_{1 \cdots t}, m\right)\left\|\mathrm{NH}\left(k_{3 \cdots t+2}, m\right)\right\| \cdots \| \mathrm{NH}\left(k_{2 n-1 \cdots t+2(n-1)}, m\right)
$$

Table 2. Performance (cycles/byte) of digest, $\mathrm{MMH}$ and $\mathrm{NH}$ constructions. In each row, the length of $\mathrm{NH}$ is always twice the length of $\mathrm{MMH}$ and digest.

\begin{tabular}{|c|c|c||c|c|c||c|c|c|}
\hline \multicolumn{3}{|c||}{ digest } & \multicolumn{3}{c||}{ MMH } & \multicolumn{3}{|c|}{ NH } \\
\hline $\begin{array}{c}\text { Output } \\
\text { bitlength }\end{array}$ & $\epsilon_{c}$ & $\begin{array}{c}\text { Speed } \\
(\mathrm{cpb})\end{array}$ & $\begin{array}{c}\text { Output } \\
\text { bitlength }\end{array}$ & $\epsilon_{c}$ & $\begin{array}{c}\text { Speed } \\
(\mathrm{cpb})\end{array}$ & $\begin{array}{c}\text { Output } \\
\text { bitlength }\end{array}$ & $\epsilon_{c}$ & $\begin{array}{c}\text { Speed } \\
(\mathrm{cpb})\end{array}$ \\
\hline 32 & $2 \times 2^{-32}$ & 0.53 & 32 & $6 \times 2^{-32}$ & 0.31 & 64 & $2^{-32}$ & 0.23 \\
64 & $2^{2} \times 2^{-64}$ & 1.05 & 64 & $6^{2} \times 2^{-64}$ & 0.57 & 128 & $2^{-64}$ & 0.39 \\
96 & $2^{3} \times 2^{-96}$ & 1.54 & 96 & $6^{3} \times 2^{-96}$ & 0.76 & 192 & $2^{-96}$ & 0.62 \\
160 & $2^{5} \times 2^{-160}$ & 2.13 & 160 & $6^{5} \times 2^{-160}$ & 1.37 & 320 & $2^{-160}$ & 1.15 \\
256 & $2^{8} \times 2^{-256}$ & 3.44 & 256 & $6^{8} \times 2^{-256}$ & 2.31 & 512 & $2^{-256}$ & 1.90 \\
\hline
\end{tabular}

\subsection{Implementations of MMH, NH and Digest Constructions}

We have tested the implementations of $\operatorname{digest}(), \mathrm{MMH}, \mathrm{NH}$ as well as their multiple-word output versions on a workstation with a $1 \mathrm{GHz}$ AMD Athlon(tm) 64 X2 Dual Core Processor (4600+ or 512 KB caches) running the 2.6.30 Linux kernel. All source codes were written in C making use of GCC 4.4.1 compiler. The number of cycles elapsed during execution was measured by the clock() instruction in the normal way (as in UMAC [25]) in our C implementations [1.

For comparison, we recompiled publicly available source codes for SHA-256 and SHA-512 22] whose reported speeds on our workstation are $12.35 \mathrm{cpb}$ and $8.54 \mathrm{cpb}$ respectively.

For application of these primitives in MACs, normally a tree hash structure is used to significantly reduce the length of universal hash key. The downsize of this method is that the resulting collision probability is not constant but proportional to the depth of the tree. For this reason, previously reported speeds for $\mathrm{MMH}$ and $\mathrm{NH}$ [38] and our results do not include the cost of key generation.

Table 2 shows the results of the experiments, which were averaged over a large number of random data inputs of at least 8 kilobytes. The speeds are in cycles/byte or cpb. Our digest constructions, at the cost of higher security, are slightly slower than MMH and NH due to extra multiplications, but still considerably faster than standard cryptographic hash functions SHA-256/512. 


\subsection{Polynomial Universal Hashing Schemes}

In this section, we will study another well-studied class of UHF based on polynomial over finite fields, including GHASH within Galois Counter Mode, PolyP, PolyQ and PolyR [12.

For simplicity, we will give a simple version of polynomial universal hashing that is the core of PolyP, PolyQ, PolyR and GHASH. Let the set of all messages be $\left\{m=\left\langle m_{1}, \ldots, m_{t}\right\rangle ; m_{i} \in \mathbb{F}_{p}\right\}$, here $p$ is the largest prime number less than $2^{b}$ and the message length is $M=t b$ bits. For any key $k \in \mathbb{F}_{p}$, we define:

$$
\operatorname{Poly}(k, m)=m_{1}+m_{2} k+m_{3} k^{2}+\cdots+m_{t} k^{t-1} \quad(\bmod p)
$$

Such a scheme does have two nice properties as follows

- The key length of the $b$-bit output Poly () scheme is fixed at $b$ bits regardless of the message length.

- Poly() provides collision resistance for both equal and unequal length messages. Suppose that the bit lengths of two different messages $m$ and $m^{\prime}$ are $b t$ and $b t^{\prime}$, then the collision probability is $\max \left\{t-1, t^{\prime}-1\right\} / p$. On the other hand, $\mathrm{MMH}, \mathrm{NH}$ and digest() only ensure collision resistance for equal length data, but not unequal length messages. The latter is intuitively because unequal length messages in $\operatorname{digest}(), \mathrm{MMH}$ and $\mathrm{NH}$ require unequal length keys, which make them incomparable for collision analysis.

The main disadvantage of a polynomial universal hashing scheme is that its security bound depends on the message length, which is the opposite of $\mathrm{MMH}$, $\mathrm{NH}$ and digest(). Namely, the collision probability of Poly () is $\epsilon=(t-1) 2^{-b}$ that is no where near the level of security obtained by our digest function when message is of a significant size. The security downside of polynomial universal hash functions does have a negative impact on their use in manual authentication where short-output but highly secure universal hash functions are required.

\section{Short-Output Universal Hash Functions in Manual Authentication Protocols}

In addition to MAC schemes, short-output universal hash functions have found use in manual authentication protocols where parties $A$ and $B$ want to authenticate their public data $m_{A / B}$ to each other without the need for passwords, shared private keys as in MACs, or pre-established security infrastructures such as a PKI. Instead authentication is bootstrapped from human trust and interactions.

Using notation taken from authors' work [17/1819 the $N$-indexed arrow $\left(\longrightarrow_{N}\right)$ indicates an unreliable and high-bandwidth (or normal) link where messages can be maliciously altered, whereas the $E$-indexed arrow $\left(\longrightarrow_{E}\right)$ represents an authentic and unspoofable (or empirical) channel. The latter is not a private channel (anyone can overhear it) and it is usually very low-bandwidth since it is implemented by humans, e.g., human conversations or manual data transfers between devices. hash () is a cryptographic hash function. Long random keys 
$k_{A / B}$ are generated by $A / B$, and $k_{A}$ is kept secret until after $k_{B}$ is revealed in Message 2. Operators $\|$ and $\oplus$ denote bitwise concatenation and exclusive-or.

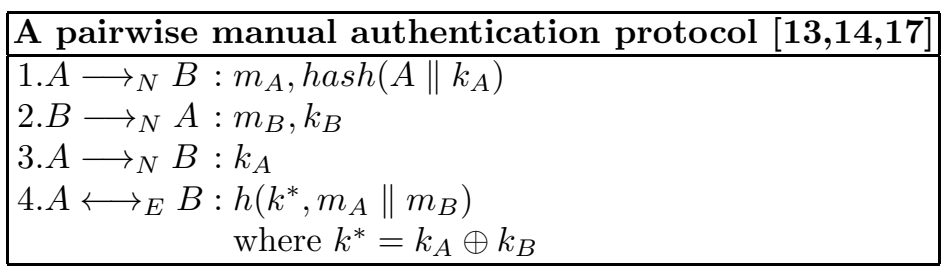

To ensure devices agree on the same data $m_{A} \| m_{b}$, their human owners manually compare the universal hash in Message 4. As human interactions are expensive, the universal hash function needs to have a short output of $b \in[16,32]$ bits.

As seen from the above protocol, the universal hash key $k^{*}$ always varies randomly and uniformly from one to another protocol run. In other words, no value of $k^{*}$ is used to hash more than one message because $k_{A / B}$ instrumental in the computation of $k^{*}$ are randomly chosen in each protocol run. This is fundamentally different from MACs which often use the same private key to hash multiple messages for a period of time 6 and hence attacks which rely on the reuse of a single private key in multiple sessions are irrelevant in manual authentication protocols. What we then want to understand is the collision and distribution properties of the universal hash function. We stress that this analysis is also applicable to group manual authentication protocols [17/18 19 26].

Should digest(), MMH or NH be used directly in Message 4 of the above protocol, random and fresh keys $k_{A / B}$ of similar size as $m_{A} \| m_{B}$ must be generated whenever the protocol is run. Obviously one can generate a long random key stream from a short seed via a pseudo-random number generator, but it can be computationally expensive especially when the authenticated data $m_{A / B}$ are of a significant size. Of course we can use one of the polynomial universal hashing functions (e.g. PolyP32, PolyQ32 or PolyR16_32 all defined in [12]) which require a short key. But since humans only can compare short value over the empirical channel, it is intolerable that the security bound of the universal hash function degrades linearly along with the length of data being authenticated.

One possibility suggested in [7/20] is to truncate the output of a cryptographic hash function to the $b$ least significant bits:

$$
h(k, m)=\operatorname{trunc}_{b}(h a s h(k \| m))
$$

Although it can be computationally infeasible to search for a full cryptographic hash collision, it is not clear whether the truncated solution is sufficiently secure because the definition of a hash function does not normally specify the distribution of individual groups of bits.

What we therefore propose is a combination of cryptographic hashing and short-output universal hash functions. Without loss of generality, we use our

${ }^{6}$ Even when a new universal hash key is generated every time a message is hashed in MAC as recommended by Handschuh and Preneel [9], the long key is stilled derived from the same private and much shorter seed or key shared between the parties. 
digest method in the following construction which is also applicable to $\mathrm{MMH}$ and NH. Let hash() be a $B$-bit cryptotgraphic hash function, e.g. SHA-2/3. First the input key is split into two parts of unequal lengths $k=k_{1} \| k_{2}$, where $k_{1}$ is $B+b$ bits and $k_{2}$ is at least 80 bits. Then our modified construction digest ${ }^{\prime}()$ which takes an arbitrarily length message $m$ is computed as follows 7

$$
\operatorname{digest}^{\prime}(k, m)=\operatorname{digest}\left(k_{1}, \operatorname{hash}\left(m \| k_{2}\right)\right)
$$

We denotes $\theta_{c}$ the hash collision probability of hash(), and it should be clear that $\theta_{c} \gg 2^{-b}$ given that $b \in[16,32]$. The following theorem shows that this construction essentially preserves both the collision and distribution security bounds, and at the same time removes the restriction on equal length input messages because the hash function hash() always produces a fixed length value.

Theorem 4. digest $^{\prime}()$ satisfies Definition 3 with the distribution probability $\epsilon_{d}=2^{-b}$ and the collision probability $\epsilon_{c}=2^{1-b}+\theta_{c}$.

Proof. Let $l_{1}$ and $l_{2}$ denote the bitlengths of keys $k_{1}$ and $k_{2}$ respectively.

We first consider collision property of digest' () . For any pair of distinct messages $m$ and $m^{\prime}$, as key $k_{2}$ varies uniformly and randomly the probability that $\operatorname{hash}\left(m \| k_{2}\right)=\operatorname{hash}\left(m^{\prime} \| k_{2}\right)$ is $\theta_{c}$. So there are two possibilities:

- When $\operatorname{hash}\left(m \| k_{2}\right)=\operatorname{hash}\left(m^{\prime} \| k_{2}\right)$ then $\operatorname{digest}\left(k_{1}, \operatorname{hash}\left(m \| k_{2}\right)\right)=$ $\operatorname{digest}\left(k_{1}, \operatorname{hash}\left(m^{\prime} \| k_{2}\right)\right)$ for any key $k_{1} \in\{0,1\}^{l_{1}}$.

- When hash $\left(m \| k_{2}\right) \neq \operatorname{hash}\left(m^{\prime} \| k_{2}\right)$ then $\operatorname{digest}\left(k_{1}, \operatorname{hash}\left(m \| k_{2}\right)\right)=$ $\operatorname{digest}\left(k_{1}\right.$, hash $\left.\left(m^{\prime} \| k_{2}\right)\right)$ with probability $2^{1-b}$.

Consequently the collision probability of $\operatorname{digest}^{\prime}()$ is

$$
\theta_{c}+\left(1-\theta_{c}\right) 2^{1-b}<\theta_{c}+2^{1-b}
$$

As regards distribution probability of digest $^{\prime}()$, we fix message $m$ of arbitrarily length and a $b$-bit value $y$ in our analysis. For each value of $k_{2}$, there will be at most $2^{l_{1}-b}$ different keys $k_{1}$ such that $\operatorname{digest}\left(k_{1}, \operatorname{hash}\left(m \| k_{2}\right)\right)=y$. Since there are $2^{l_{2}}$ different keys $k_{2}$, there will be at most $2^{l_{1}-b} 2^{l_{2}}=2^{l_{1}+l_{2}-b}$ different pairs $\left(k_{1}, k_{2}\right)$ or different keys $k$ such that $\operatorname{digest}\left(k_{1}, \operatorname{hash}\left(m \| k_{2}\right)\right)=y$. The distribution probability of digest $^{\prime}()$ is therefore $2^{-b}$

There is another short-output universal hash function called UHASH16 (and also UHASH32) of Krovetz [3] with 16-bit output and $\epsilon_{c} \approx 2^{-15}$. The universal hash key length is fixed at around $2^{14}$ bits or $2 \mathrm{~KB}$ that is much longer than $B+b+80$ of digest $^{\prime}($ ), but UHASH16 has the advantage of being more efficient than a cryptographic hash function required in digest $^{\prime}()$.

UHASH16 is the result of a rather non-trivial combination (and tree hash structure) of NH and polynomial universal hashing. First, NH is used to compress

7 The concatenation of $m$ and $k_{2}$ is hashed to make it much harder for the intruder to search for collision because a large number of bits of the hash input will not be controlled by the intruder. 
input messages into strings which are typically many times (e.g. 512 times in UHASH16) shorter than the original input message. Second, the compressed message is hashed with a polynomial universal hash function into a 128-bit string. Finally, the 128-bit string is hashed using an inner-product hash into a 16-bit string. We should point out that there is a trade-off between key length and computational efficiency, i.e. the shorter is the NH key the more computation is required for polynomial hashing in the second stage.

Acknowledgements. Nguyen's work on this paper was supported by a research grant from the US Office of Naval Research. Roscoe's was partially supported by funding from the US Office of Naval Research.

The authors would like to thank Dr. Andrew Ker at Oxford University for his help with statistical analysis of the digest constructions.

Progresses on the security proof of our digest functions were first made when Nguyen visited Professor Bart Preneel and Dr. Frederik Vercauteren at the Computer Security and Industrial Cryptography (COSIC) research group at the Katholieke Universiteit Leuven in September and October 2010. The authors would like to thank them for their time and support as well as Drs Nicky Mouha and Antoon Bosselaers at COSIC for pointing out the relevance of the multiplicative universal hashing scheme of Dietzfelbinger et al. [5] and the literature on hash function implementation and speed measurement for benchmarking.

We also received helpful comments from many anonymous referees as well as had fruitful discussions and technical feedbacks from Professor Serge Vaudenay and Dr. Atefeh Mashatan when Nguyen visited the Security and Cryptography Laboratory (LASEC) at the Swiss Federal Institute of Technologies (EPFL) in February and March 2011. The feedbacks significantly improve the technical quality and presentation of the paper.

\section{References}

1. http://www.cs.ox.ac.uk/publications/publication5935-abstract.html

2. http://software.intel.com/en-us/articles/ carry-less-multiplication-and-its-usage-for-computing-the-gcm-mode/

3. Black, J., Halevi, S., Krawczyk, H., Krovetz, T., Rogaway, P.: UMAC: Fast and Secure Message Authentication. In: Wiener, M. (ed.) CRYPTO 1999. LNCS, vol. 1666, pp. 216-233. Springer, Heidelberg (1999)

4. Carter, J.L., Wegman, M.N.: Universal Classes of Hash Functions. Journal of Computer and System Sciences 18, 143-154 (1979)

5. Dietzfelbinger, M., Hagerup, T., Katajainen, J., Penttonen, M.: A reliable randomized algorithm for the closest-pair problem. Journal Algorithms 25, 19-51 (1997)

6. Etzel, M., Patel, S., Ramzan, Z.: SQUARE HASH: Fast Message Authentication via Optimized Universal Hash Functions. In: Wiener, M. (ed.) CRYPTO 1999. LNCS, vol. 1666, pp. 234-251. Springer, Heidelberg (1999)

7. Gehrmann, C., Mitchell, C., Nyberg, K.: Manual Authentication for Wireless Devices. RSA Cryptobytes 7(1), 29-37 (2004)

8. Halevi, S., Krawczyk, H.: MMH: Software Message Authentication in the Gbit/Second Rates. In: Biham, E. (ed.) FSE 1997. LNCS, vol. 1267, pp. 172-189. Springer, Heidelberg (1997) 
9. Handschuh, H., Preneel, B.: Key-Recovery Attacks on Universal Hash Function Based MAC Algorithms. In: Wagner, D. (ed.) CRYPTO 2008. LNCS, vol. 5157, pp. 144-161. Springer, Heidelberg (2008)

10. Nguyen, L.H. (ed.): Information Technology - Security Techniques - Entity authentication - Part 6: Mechanisms using manual data transfer, ISO/IEC 9798-6 (2010)

11. Krawczyk, H.: New Hash Functions for Message Authentication. In: Guillou, L.C., Quisquater, J.-J. (eds.) EUROCRYPT 1995. LNCS, vol. 921, pp. 301-310. Springer, Heidelberg (1995)

12. Krovetz, T., Rogaway, P.: Fast Universal Hashing with Small Keys and No Preprocessing: The PolyR Construction. In: Won, D. (ed.) ICISC 2000. LNCS, vol. 2015, pp. 73-89. Springer, Heidelberg (2001)

13. Laur, S., Nyberg, K.: Efficient Mutual Data Authentication Using Manually Authenticated Strings. In: Pointcheval, D., Mu, Y., Chen, K. (eds.) CANS 2006. LNCS, vol. 4301, pp. 90-107. Springer, Heidelberg (2006)

14. Lindell, A.Y.: Comparison-Based Key Exchange and the Security of the Numeric Comparison Mode in Bluetooth v2.1. In: Fischlin, M. (ed.) CT-RSA 2009. LNCS, vol. 5473, pp. 66-83. Springer, Heidelberg (2009)

15. Mansour, Y., Nisan, N., Tiwari, P.: The Computational Complexity of Universal Hashing. In: ACM STOC, pp. 235-243 (1990)

16. Mashatan, A., Stinson, D.: Practical Unconditionally Secure Two-channel Message Authentication. Designs, Codes and Cryptography 55, 169-188 (2010)

17. Nguyen, L.H., Roscoe, A.W.: Authentication protocols based on low-bandwidth unspoofable channels: A comparative survey. Journal of Computer Security 19(1), 139-201 (2011)

18. Nguyen, L.H., Roscoe, A.W.: Efficient group authentication protocol based on human interaction. In: FCS-ARSPA, pp. 9-31 (2006)

19. Nguyen, L.H., Roscoe, A.W.: Authenticating ad-hoc networks by comparison of short digests. Information and Computation 206(2-4), 250-271 (2008)

20. Pasini, S., Vaudenay, S.: SAS-Based Authenticated Key Agreement. In: Yung, M., Dodis, Y., Kiayias, A., Malkin, T. (eds.) PKC 2006. LNCS, vol. 3958, pp. 395-409. Springer, Heidelberg (2006)

21. Roscoe, A.W., Nguyen, L.H.: Security in computing networks. World Intellectual Property Organization. Application number: PCT/GB2006/004113. Publication number: WO/2007/052045. Filed on November 03, 2006. Publication date, May 10 (2007)

22. http://www . aarongifford.com/computers/sha.html

23. Shamir, A.: SQUASH - A New MAC with Provable Security Properties for Highly Constrained Devices Such as RFID Tags. In: Nyberg, K. (ed.) FSE 2008. LNCS, vol. 5086, pp. 144-157. Springer, Heidelberg (2008)

24. Stinson, D.R.: Universal Hashing and Authentication Codes. In: Feigenbaum, J. (ed.) CRYPTO 1991. LNCS, vol. 576, pp. 74-85. Springer, Heidelberg (1992)

25. The performance of UMAC can be found at, http://fastcrypto.org/umac/

26. Valkonen, J., Asokan, N., Nyberg, K.: Ad Hoc Security Associations for Groups. In: Buttyán, L., Gligor, V.D., Westhoff, D. (eds.) ESAS 2006. LNCS, vol. 4357, pp. 150-164. Springer, Heidelberg (2006) 